\title{
A CHARACTERIZATION OF M. W. WILSON'S CRITERION FOR NONNEGATIVE EXPANSIONS OF ORTHOGONAL POLYNOMIALS
}

\author{
CHARLES A. MICCHELLI
}

ABSTRACT. Given a nonnegative function $f(x)$, M. W. Wilson observed that if

$$
\int_{0}^{\infty} f(x) Q_{i}(x) Q_{j}(x) d \mu(x)<0, \quad i \neq j,
$$

then the polynomials $P_{n}(x), P_{n}(0)=1$, orthogonal relative to $f(x) d \mu(x)$, have an expansion

$$
P_{n}(x)=\sum_{k=0}^{n} a_{k n} Q_{k}(x)
$$

with nonnegative coefficients $a_{k n} \geqslant 0$ where $Q_{n}(x), Q_{n}(0)=1$, are orthogonal relative to $d \mu(x)$. Recently it was shown that (1) holds for $f(x)=x^{c}, 0<c<1$. In this paper we characterize those functions $f(x)$ for which (1) is valid for all positive measures $d \mu(x)$.

Let $d \mu(x)$ be a positive measure on $[0, \infty)$ with $\mu(x)$ having an infinite number of points of increase. Suppose also that $d \mu(x)$ has finite moments of all orders and $Q_{n}(x), n=0,1, \ldots$, are the associated orthogonal polynomials normalized so that $Q_{n}(0)=1$. Given a nonnegative function $f(x)$, M. W. Wilson [5] observed that if

$$
\int_{0}^{\infty} f(x) Q_{i}(x) Q_{j}(x) d \mu(x) \leqslant 0, \quad i \neq j,
$$

then the polynomials $P_{n}(x), P_{n}(0)=1$, orthogonal relative to $f(x) d \mu(x)$ have an expansion

$$
P_{n}(x)=\sum_{k=0}^{n} a_{k n} Q_{k}(x)
$$

with nonnegative coefficients, $a_{k n} \geqslant 0$. Recently, W. Trench [3] showed that (1) holds for $f(x)=x^{c}, 0<c<1$, thus establishing a conjecture made by Askey [1]. In this paper we characterize those functions $f(x)$ for which (1) is valid for all positive measures $d \mu(x)$.

THEOREM. Let $d \mu(x), Q_{n}(x), n=0,1,2, \ldots$, and $f(x)$ be as described above. Then

$$
\int_{0}^{\infty} f(x) Q_{i}(x) Q_{j}(x) d \mu(x) \leqslant 0, \quad i \neq j
$$

Received by the editors August 24, 1977.

AMS (MOS) subject classifications (1970). Primary 42A52. 
if and only if $f^{\prime}(x)$ is completely monotonic on $[0, \infty)$.

Proof. First we prove the necessity of this condition on $f(x)$. Thus, in particular, we have that

$$
\int_{0}^{\infty} f(x) Q_{n}(x) d \mu(x) \leqslant 0, \quad n \geqslant 1 .
$$

Fix $0<x_{1}<\cdots<x_{n+1}$ and let $d \nu(x)$ be any positive measure supported on $x_{1}, \ldots, x_{n+1}$. Then by a straightforward limiting argument we conclude from (2) (which holds for all $d \mu$ with $\mu(x)$ having an infinite number of points of increase) that

$$
\int_{0}^{\infty} f(x) Q_{n}(x) d \nu(x) \leqslant 0 .
$$

Let $p(x)$ be the polynomial of degree $n$ which interpolates $f$ at $x_{1}, \ldots, x_{n+1}$. Then $f(x)=p(x)$ a.e. $d \nu(x)$ and consequently

$$
\begin{aligned}
\int_{0}^{\infty} f(x) Q_{n}(x) d \nu(x) & =f\left[x_{1}, \ldots, x_{n+1}\right] \int_{0}^{\infty} x^{n} Q_{n}(x) d \nu(x) \\
& =q_{n}^{-1} f\left[x_{1}, \ldots, x_{n+1}\right] \int_{0}^{\infty} Q_{n}^{2}(x) d \nu(x)
\end{aligned}
$$

where $Q_{n}(x)=q_{n} x^{n}+\cdots$ and $f\left[x_{1}, \ldots, x_{n+1}\right]$ is the divided difference of $f(x)$ at $x_{1}, \ldots, x_{n+1}$. Since $Q_{n}(0)=1$, it follows that $\operatorname{sgn} q_{n}=(-1)^{n}$ and hence

$$
(-1)^{n+1} f\left[x_{1}, \ldots, x_{n+1}\right] \geqslant 0, \quad n \geqslant 1 .
$$

Thus $(-1)^{n} f^{(n+1)}(x) \geqslant 0$ and hence according to Widder [3], $f^{\prime}(x)$ is completely monotonic on $[0, \infty)$.

Conversley, let $f^{\prime}(x)$ be completely monotonic. Then $f(x)=O(x)$ as $x \rightarrow$ $\infty$ and so the integrals in (1) are finite. Choose an $a>0$ and suppose $Q_{n}(x ; a), n=0,1,2, \ldots$, are the polynomials orthogonal to $d \mu(x)$ on $[0, a]$

$$
\begin{gathered}
\int_{0}^{a} Q_{i}(x ; a) Q_{j}(x ; a) d \mu(x)=0, \quad i \neq j, \\
Q_{i}(0 ; a)=1 .
\end{gathered}
$$

Then clearly $\lim _{a \rightarrow \infty} Q_{i}(x ; a)=Q_{i}(x), x \geqslant 0$, uniformly on bounded intervals. Hence to prove (1) it is sufficient to demonstrate that

$$
\int_{0}^{a} f(x) Q_{i}(x ; a) Q_{j}(x ; a) d \mu(x) \leqslant 0, \quad i \neq j .
$$

For convenience we suppress the $a$ when writing $Q_{i}(x ; a)$ and let it be understood that these polynomials are orthogonal with respect to $d \mu(x)$ over $[0, a] . Q_{n}(x)$ satisfies a three term recurrence relation

$$
\begin{aligned}
&-x Q_{n}(x)=\mu_{n} Q_{n-1}(x)-\left(\lambda_{n}+\mu_{n}\right) Q_{n}(x)+\lambda_{n} Q_{n+1}(x) \\
& \mu_{0}=0, \mu_{n+1}>0, \lambda_{n}>0, n \geqslant 0,
\end{aligned}
$$

and since $Q_{n}(0)=1$ 


$$
\int_{0}^{a} Q_{i}(x) Q_{j}(x) d \mu(x)=\delta_{i j} \pi_{j}^{-1}
$$

where $\pi_{0}=1, \pi_{j}=\lambda_{0} \lambda_{1} \cdots \lambda_{j-1} / \mu_{1} \mu_{2} \cdots \mu_{j}, j \geqslant 1$.

Consequently, $\hat{Q}_{i}=\pi_{i}^{1 / 2} Q_{i}$ are orthonormal and according to (3)

$$
A_{i j}:=\int_{0}^{a} x \hat{Q}_{i}(x) \hat{Q}_{j}(x) d \mu(x) \leqslant 0, \quad i \neq j .
$$

Now, fix a positive integer $p$ and consider the $p+1 \times p+1$ section of the infinite matrix $A$ defined above,

$$
\left(A_{p}\right)_{i j}=\int_{0}^{a} x \hat{Q}_{i}(x) \hat{Q}_{j}(x) d \mu(x), \quad 0 \leqslant i, j \leqslant p .
$$

$A_{p}$ is a Stieltjes matrix, because it is obviously symmetric and positive definite and according to (4) has nonpositive off diagonal elements.

This matrix has an easily derived spectral decomposition. In fact, if $0<x_{1}<\cdots<x_{p+1}$ are the zeros of $Q_{p+1}(x)$, then Gaussian quadrature for $d \mu(x)$ on $[0, a]$ gives

$$
\int_{0}^{a} F(x) d \mu(x)=\sum_{k=1}^{p+1} w_{k} F\left(x_{k}\right), \quad F \in P_{2 p+1},
$$

$P_{2 p+1}=$ polynomials of degree $\leqslant 2 p+1$. Using the Christoffel Darboux formula

$$
(x-y) \sum_{k=0}^{n} Q_{k}(x) Q_{k}(y) \pi_{k}=\lambda_{n} \pi_{n}\left(Q_{n}(x) Q_{n+1}(y)-Q_{n}(y) Q_{n+1}(x)\right)
$$

a straightforward calculation shows that

$$
\sum_{k=1}^{p+1} Q_{k}\left(x_{l}\right) Q_{k}\left(x_{m}\right) \pi_{k}=\delta_{l m} w_{l}^{-1}, \quad l, m \leqslant p .
$$

Hence the $p+1$-dimensional vectors $u_{l}=\left(w_{l}^{1 / 2} \hat{Q}_{0}\left(x_{l}\right), \ldots, w_{l}^{1 / 2} \hat{Q}_{p}\left(x_{l}\right)\right)^{T}$ are orthonormal,

$$
\left(u_{l}, u_{m}\right)=\delta_{l m}, \quad l, m \leqslant p .
$$

Applying the Gaussian quadrature formula $(5)$ to $F(x)=x \hat{Q}_{i}(x) \hat{Q}_{j}(x)$ gives us the desired representation for $A_{p}$,

$$
A_{p}=\sum_{l=1}^{p+1} x_{l} u_{l} u_{l}^{T}
$$

Now, we are ready to use the fact that $f^{\prime}(x)$ is completely monotonic. This property of $f$ implies that

$$
f(x)=f\left(x_{p+1}\right)-\sum_{m=0}^{\infty} \frac{(-1)^{m+1} f^{(m)}\left(x_{p+1}\right)}{m !}\left(x_{p+1}-x\right)^{m}
$$

and thus

$$
f\left(A_{p}\right)=f\left(x_{p+1}\right) I-\sum_{m=1}^{\infty} \frac{(-1)^{m+1}}{m !} f^{(m)}\left(x_{p+1}\right)\left(x_{p+1} I-A_{p}\right)^{m} .
$$


The matrix $x_{p+1} I-A_{p}$ is positive definite, symmetric and has all nonnegative elements. Therefore (7) shows that $f\left(A_{p}\right)$ is also a Stieltjes matrix (for a discussion of functions which preserve the class of Stieltjes matrices see [2]). Consequently, we have shown that for all $p$

$$
f\left(A_{p}\right)_{i j} \leqslant 0, \quad i \neq j, i, j \leqslant p .
$$

The spectral decomposition (6) of $A_{p}$ gives us an alternate expression for $f\left(A_{p}\right)$. Indeed (6) implies

$$
f\left(A_{p}\right)=\sum_{l=1}^{p+1} f\left(x_{l}\right) u_{l} u_{l}^{T}
$$

or equivalently

$$
f\left(A_{p}\right)_{i j}=\sum_{l=1}^{p+1} f\left(x_{l}\right) w_{l} \hat{Q}_{i}\left(x_{l}\right) \hat{Q}_{j}\left(x_{l}\right) .
$$

Letting $p \rightarrow \infty$ and noting that the right hand side of (9) is the result of applying the Gaussian rule (5) to $F=f \hat{Q}_{i} \hat{Q}_{j}$ we obtain

$$
0 \geqslant \lim _{p \rightarrow \infty} f\left(A_{p}\right)_{i j}=\int_{0}^{a} f(x) \hat{Q}_{i}(x) \hat{Q}_{j}(x) d \mu(x), \quad i \neq j .
$$

This assertion completes the proof of the theorem.

\section{REFERENCES}

1. R. Askey, Orthogonal expansions with positive coefficients, Proc. Amer. Math. Soc. 16 (1965), 1191-1194.

2. C. A. Micchelli and R. A. Willoughby, On functions which preserve the class of Stieltjes matrices, Linear Algebra and Appl. (to appear).

3. William F. Trench, Proof of a conjecture of Askey on orthogonal expansions with positive coefficients, Bull. Amer. Math. Soc. 81 (1975), 954-956.

4. D. V. Widder, The Laplace Transform, Princeton Univ. Press, Princeton, N.J., 1946.

5. M. W. Wilson, Nonnegative expansions of polynomials, Proc. Amer. Math. Soc. 24 (1970), 100-102.

IBM T. J. Watson Research Center, Yorktown Heights, New York 10598 